Cahiers $d u$ MONDE RUSSE

\section{Cahiers du monde russe}

Russie - Empire russe - Union soviétique et États indépendants

\title{
Adele Marie Barker, Jehanne M. Gheith, eds., A history of women's writing in Russia
}

\section{Michel Aucouturier}

\section{OpenEdition \\ Journals}

Édition électronique

URL : https://journals.openedition.org/monderusse/4151

DOl : 10.4000/monderusse.4151

ISSN : $1777-5388$

\section{Éditeur}

Éditions de l'EHESS

\section{Édition imprimée}

Date de publication : 1 octobre 2003

Pagination : 816-819

ISBN : 2-7132-1833-0

ISSN : $1252-6576$

Référence électronique

Michel Aucouturier, « Adele Marie Barker, Jehanne M. Gheith, eds., A history of women's writing in Russia », Cahiers du monde russe [En ligne], 44/4 | 2003, mis en ligne le 19 juin 2009, consulté le 02 septembre 2022. URL : http://journals.openedition.org/monderusse/4151 ; DOI : https://doi.org/ $10.4000 /$ monderusse.4151

Ce document a été généré automatiquement le 2 septembre 2022.

Tous droits réservés 


\title{
Adele Marie Barker, Jehanne M. Gheith, eds., A history of women's writing in Russia
}

\author{
Michel Aucouturier
}

\section{RÉFÉRENCE}

Adele Marie BARKER, Jehanne M. GHEITH, eds., A history of women's writing in

Russia. Cambridge-New York, Cambridge University Press, 391 p.

1 L'expression anglaise women's writing, dont la traduction la plus exacte serait « les écrits des femmes ", permet d'éviter les notions problématiques d'« écriture féminine " (à laquelle renvoient, sans s'y rallier explicitement, certains des auteurs de ce recueil) ou de "littérature féminine", aussi artificielle et inopérante que, par exemple, celle de «littérature prolétarienne» ou de "littérature juive russe». Les auteurs du recueil reconnaissent généralement que les œuvres écrites par les femmes, même si elles peuvent témoigner d'une sensibilité particulière et d'une prédilection pour certains thèmes (en particulier celui de la condition féminine et de l'émancipation de la femme), même si, dans le domaine poétique, elles ont pu créer un "personnage d'auteur" explicitement féminin, se situent dans le même champ littéraire, ont généralement les mêmes lecteurs, éditeurs, critiques, et se nourrissent des mêmes sources et des mêmes influences que celles des hommes.

2 Le terme women's writing a l'avantage et l'inconvénient de l'empirisme : il évite les partis pris théoriques, mais définit trop largement son objet pour qu'on puisse parler d'une véritable " histoire ». En fait, nous avons affaire à une série discontinue d'études qui ne couvrent pas de façon uniforme dix siècles d'histoire de la littérature russe, mais y découpent quinze sujets d'ampleur variée.

3 La première (de Rosalind McKenzie) est en fait extérieure au thème du livre puisque, consacrée à la littérature médiévale, elle ne traite pas des écrits des femmes, mais de leur 
image. C'est seulement dans les deux suivantes, qui couvrent le xvIII et le début du $\mathrm{xIX}^{\mathrm{e}}$ siècle, qu'apparaissent les premières figures de femmes-écrivains : Catriona Kelly («Sappho, Corinna and Niobe: genres and personae in Russian women's writing, 1760-1820 ») aborde leur autoreprésentation stylisée à partir d'une étude socio-culturelle de l'éducation des femmes et de leur place dans la société russe du XVIII siècle. Judith Vowles (« The inexperienced muse: Russian women and poetry in the first half of the nineteenth century ») fait apparaître le lien qui unit leur percée dans le domaine poétique à la sensibilité et à l'esthétique du préromantisme et du romantisme.

4 Seule une étude est consacrée à la prose du XIX ${ }^{e}$ siècle proprement dite : celle de Jehanne M. Gheith, "Women of the 1830s and 1850s: alternative periods》. Consacrée à la contribution féminine à la prose réaliste du milieu du siècle, elle décèle dans sa thématique propre une continuité qui remet en question l'opposition traditionnelle entre la génération des années 1840 et celle des années 1860. L'article de Mary Zirin (« “A particle of ourself" : pre-Revolutionary autobiography by Russian women writers ») embrasse un champ chronologique qui s'étend au-delà du $\mathrm{XIX}^{\mathrm{e}}$ siècle : à travers huit exemples, échelonnées de 1836 (Nadežda Durova, la fameuse kavalerist-devica saluée par Puškin) à 1971 (Anastasija Cvetaeva, la sœur de Marina), l'auteur essaie de saisir la spécificité de l'autobiographie féminine, exprimant l'image que les femmes se font de leur condition.

5 Des deux articles consacrés à la période intermédiaire entre le $\mathrm{xIX}^{\mathrm{e}}$ et le $\mathrm{xx}^{\mathrm{e}}$ siècle, le premier, celui de Jenifer Presto ("Women in Russian symbolism : beyond the algebra of love») se propose de mettre en évidence le rôle que les femmes ont joué en tant que "sujets", et non simplement "objets", dans la poésie symboliste. La longue étude de Rosalind Marsh («Realist prose writers, 1881-1929») embrasse, comme celle de Mary Zirin, un champ chronologique peu homogène : à cheval sur deux périodes historiques nettement différenciées, elle passe en revue un vaste corpus, y mettant en relief les textes « réalistes » et les textes « féministes ».

$6 \mathrm{Au} \mathrm{Xx}^{\mathrm{e}}$ siècle où, en particulier du fait de la révolution, l'émancipation politique, sociale et culturelle de la femme a fait des progrès considérables, est consacrée à juste titre plus de la moitié du recueil. Des deux études portant sur la littérature de l'émigration, la plus originale est celle d'Olga Bakich et Carol Ueland, «The Eastern path of exile : Russian women's writing in China », qui montre le rôle important des femmes dans un secteur exotique et encore peu exploré de la littérature de l'émigration. Celle de Catherine Ciepiela, en revanche («The women of Russian Montparnasse, Paris 1920-1940») n'apporte qu'une présentation nouvelle de faits bien connus.

7 Six études portent sur la littérature soviétique. Celle de Katherine Hodgson ("Women and gender in post-symbolist poetry and the Stalin era ») replace les œuvres de Cvetaeva et d'Ahmatova dans le cadre d'une génération poétique féminine dont l'essor a été brisé par la révolution qui en a condamné une partie à l'exil et une autre au silence - dont elles n'ont émergé peu à peu qu'après la mort de Stalin.

Beth Holmgren, dont l'étude consacrée aux femmes-écrivains de la période de l'aprèsguerre et du dégel (« Writing the female body politic, 1945-1985») parle non sans raison d'une "féminisation de la littérature soviétique des décennies de l'après-guerre, par contraste et en réaction au mythe des années 1920 d'une nouvelle société masculinisée ", présente une thèse intéressante. Elle voit dans les œuvres de Vera Panova, Galina Nikolaeva, Antonina Koptjaeva, puis Natal'ja Baranskaja et I. Grekova, l'affirmation d'un "corps politique féminin", incarnant une nouvelle modalité de l'homme soviétique, 
enrichie de valeurs (que l'on qualifie parfois trop rapidement de " petites-bourgeoises ", et que l'auteur préfère définir par le terme de middlebrow, " moyennes »- c'est-à-dire intermédiaires entre l'élitiste et le populaire), grâce à l'importance sociale acquise par les femmes après la révolution.

En relevant les mêmes valeurs dans l'œuvre d'écrivains clandestins ou dissidents, comme Evgenija Ginzburg, Lidija Čukovskaja, Lidija Ginzburg, ou Julija Voznesenskaja, l'auteur relativise le facteur proprement politique (ou institutionnel) dans l'analyse de l'évolution littéraire de la période soviétique, remettant ainsi en cause les schémas habituels de la critique occidentale. C'est ce que fait aussi Anna Krylova («In their own words : Soviet women writers and the search for self »), en dénonçant la disqualification par celle-ci des écrivains dits "officiels». Se fondant sur l'analyse d'un corpus qui recoupe partiellement celui de l'article précédent (les autobiographies, documentaires et " fictionnelles », de Vera Inber, Antonina Koptjaeva et Vera Panova), elle défend, elle aussi, une thèse qui n'a cependant rien de très novateur: les stéréotypes officiels n'empêchent pas la construction et l'expression d'un itinéraire individuel de femme et d'écrivain.

10 Les trois derniers articles, "Women's poetry since the sixties », par Stephanie Sandler, "The persistence of memory : women's prose since the sixties », par Adele Marie Barker et «Perestroika and post Soviet prose : from dazzle to dispersal » par Helena Goscilo, présentent un panorama de la littérature russe du dernier tiers du siècle, et plus particulièrement de sa dernière décennie, marquée par la perestroïka et la fin du régime soviétique. Elles mettent en évidence la place croissante qu'y occupent les femmes allant jusqu'à annexer des domaines qui étaient jusqu'alors la chasse gardée des hommes, comme le roman policier.

11 L'enjeu collectif de ce travail semble avoir été de montrer que les «écrits des femmes » occupent dans la littérature russe une place beaucoup plus importante que convenu. Son principal mérite est de tirer de l'oubli un grand nombre de figures et d'œuvres de femmes-écrivains restées en marge des synthèses courantes. Leurs bio-bibliographies succinctes sont réunies à la fin de l'ouvrage dans une liste apparemment exhaustive de plus de cinquante pages, complétée par une abondante bibliographie des travaux critiques qui leur sont consacrés. Tout cela en fait un instrument de travail indispensable, mais ne suffit pas à bouleverser les hiérarchies acquises et à remettre en question, comme le suggèrent Adele Marie Barker et Jehanne M. Gheith dans leur introduction, la périodisation traditionnelle de l'histoire de la littérature russe.

12 D'un point de vue théorique ou méthodologique, le recueil pose une grande question d'histoire littéraire, sans doute insuffisamment explorée jusqu'ici: quelles sont les conditions politiques, sociales, culturelles, purement littéraires (spécificité et signification de tel ou tel genre ou forme littéraire) qui ont déterminé le rôle que les femmes ont pu jouer dans la littérature russe à différents moments de son histoire? On a l'impression, en lisant l'ouvrage, que la revendication féministe obscurcit parfois cette question (en opposant, par exemple, les œuvres écrites par les femmes à une "tradition masculine dominante »), et empêche même de la formuler avec une netteté suffisante. Du moins a-t-il le mérite de l'avoir posée. 